\title{
COMMENTARY
}

\section{Optical properties of magnetoelectric multiferroics}

\author{
Gavin Lawes \\ Department of Physics and Astronomy, Wayne State University, Detroit, MI 48201, USA \\ glawes@wayne.edu
}

Systems exhibiting simultaneous magnetic and ferroelectric order, dubbed magnetoelectric multiferroics, have become an increasingly popular area of study over the past several years [1]. Ferroelectrics exhibit spontaneous electric polarization even in the absence of an external electric field, while magnetic materials develop long-range spin order. This magnetic order can be ferromagnetic, with the local atomic moments all oriented in the same direction, or antiferromagnetic, with adjacent local moments oriented in different directions. Both ferroelectrics and magnetic materials (especially ferromagnets) are very widely used in a number of applications. From a fundamental science standpoint, multiferroic systems provide an opportunity to study large magnetoelectric effects, that is, the ability of an electric field to produce a magnetization or, conversely, the ability of a magnetic field to produce an electric polarization. These materials not only offer a platform for investigating coupled spin and charge degrees of freedom, but are also expected to have important applications for developing new devices, including voltage switchable magnetic memory and tunable optical elements. Very generally, multiferroics can be separated into two categories: single-phase multiferroics, in which both the magnetic and ferroelectric orders develop involving the same set of atoms [2,3], and composite multiferroics, which consist of distinct ferromagnetic and ferroelectric constituents and are often structured at the nanoscale [4]. Although both classes of multiferroics exhibit novel optical properties, I will restrict this commentary to single-phase homogeneous multiferroic materials.

Within the broad class of single-phase multiferroics, the co-existing magnetic and ferroelectric orders can develop simultaneously at a single phase transition, as observed in $\mathrm{TbMnO}_{3}$ [2], or they can arise separately at distinct transitions, as observed in $\mathrm{YMnO}_{3}$ [3]. Although the detailed nature of the multiferroic state differs from system to system, there are several common features. The polar multiferroic state breaks both time reversal symmetry (due to the magnetic order) and spatial inversion symmetry (due to ferroelectric order). This has profound significance for the optical properties of these materials.

There have been a number of studies investigating the relationship between magnetism and ferroelectricity in multiferroic systems. Perhaps most remarkably, it has been found that for many of these materials, the ferroelectric polarization is exceptionally sensitive to an external magnetic field. In $\mathrm{TbMnO}_{3}$ for example, the spontaneous polarization can be switched from the $c$-axis to the $a$-axis by applying a magnetic field [2]. This magnetically switchable polarization promises considerable versatility for incorporating multiferroics in magneto-optical devices. This coupling between the ferroelectric polarization and magnetic field is also reflected in the magnetodielectric response (magnetic field induced change in dielectric properties) in multiferroics. The low-frequency relative permittivity in multiferroics often scales with an applied magnetic field, with the relative permittivity in $\mathrm{DyMnO}_{3}$ exhibiting a change of $500 \%$ in a field of $4 \mathrm{~T}$ at $18 \mathrm{~K}$ [5]. This magnetodielectric coupling is indicative of the sizeable spin-lattice coupling in many of these materials.

The control of the ferroelectric polarization by a magnetic field or of the magnetization by an electric field in multiferroics is schematically illustrated in Fig. 1. Very generally, the direction of the polarization vector is fixed by the symmetry of the magnetic structure (see, for example, Ref. 6), as indicated in Fig. 1(a). Applying an external magnetic field will rotate the magnetization, which in turn will rotate the locked polarization, as shown in Fig. 1(b).

(C)2008 Society of Photo-Optical Instrumentation Engineers [DOI: 10.1117/1.3046676] 


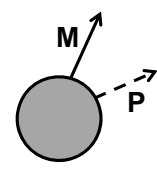

(a)

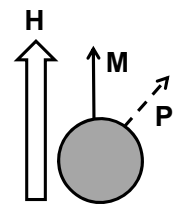

(b)

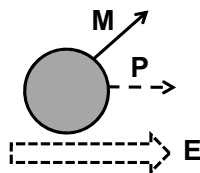

(c)

Fig. 1. (a) Schematic diagram of the magnetization and polarization vectors in a generic multiferroic material. The direction of the polarization vector is determined from the symmetry of the magnetic structure. (b) The magnetization can be rotated by an external magnetic field, effectively modifying the (locked) polarization. (c) The polarization can be rotated by an external electric field, effectively modifying the magnetization.

Conversely, applying an electric field will rotate the polarization, leading to a change in magnetization (Fig. 1(c)). In real multiferroics, the spin structure is generally more complex than the simple ferromagnetism indicated in Fig. 1, which leads to a more complicated dependence of the polarization on external magnetic fields.

The substantial spin-lattice coupling in multiferroics is reflected in the magneto-optical response in multiferroic systems. The high energy dielectric constant in $\mathrm{Ni}_{3} \mathrm{~V}_{2} \mathrm{O}_{8}$ shows a strong dependence on magnetic field in the multiferroic phase [7]. The magnetic field induced change in the relative permittivity of $\mathrm{Ni}_{3} \mathrm{~V}_{2} \mathrm{O}_{8}$ reaches $15 \%$ at $1.3 \mathrm{eV}$ in a $30 \mathrm{~T}$ magnetic field applied along the $b$-axis at $5 \mathrm{~K}$. It is believed that this enormous response near optical frequencies arises from a magnetic-field induced distortion of the local $\mathrm{NiO}_{6}$ structure in this crystal. This result highlights one of the mechanisms producing the significant magneto-optical response in multiferroics. The structural distortions associated with the changing polarization can dramatically affect the optic phonon frequencies, which, in turn, strongly modifies the high frequency response in these materials.

Multiferroic materials also exhibit remarkable optical properties in the absence of any external magnetic field. $\mathrm{YMnO}_{3}$ is a hexagonal multiferroic having a ferroelectric transition temperature close to $900 \mathrm{~K}$ and an antiferromagnetic transition near $90 \mathrm{~K}$ [3]. Although the domain structure orientation cannot be resolved using linear optics, second harmonic generation can distinguish the $180^{\circ}$ ferroelectric and antiferromagnetic domains. Measurements of the non-linear optical response in $\mathrm{YMnO}_{3}$ crystals indicate that the ferroelectric and antiferromagnetic domain walls are strongly coupled, with ferroelectric domain walls always co-existing with antiferromagnetic domain walls [3]. This provides clear evidence for the strong coupling between the magnetic and ferroelectric structures in this system, and also suggests an approach for incorporating multiferroics into optical devices. Because the second harmonic light intensity is modulated by the multiferroic domain structure, modifying the domain structure through external magnetic and/or electric fields is expected to provide a method for tuning the nonlinear optical response in these systems. This effect has been observed in $\mathrm{HoMnO}_{3}$, where the magnetic structure can be modified by an applied electric field, which in turn produces a change in the second harmonic generation signal [8]. Recently, multiferroic $\mathrm{Bi}_{2} \mathrm{FeCrO}_{6}$ has also been predicted to show an enormous change in the second harmonic generation response, of approximately $170 \%$, in an applied magnetic field [9].

There are predictions for even more striking optical properties in multiferroics. Coexisting ferromagnetic order, denoted by $\vec{M}$, and ferroelectric order, $\vec{P}$, give rise to a toroidal moment, $\vec{\tau}=\vec{P} \times \vec{M}$. It has been proposed that an inhomogeneous toroidal moment can give rise to real-space directional birefringence, which is independent of polarization [10]. Within this context, a spatial variation in the toroidal moment (as occurs, for example, across a domain 
wall) produces the optical equivalent of a Lorentz force on the light. This directional dependent optical path would lead to non-reciprocal refraction through domain walls. This effect could potentially be exploited to produce novel optical devices, as the domain wall structure, hence optical response, could be switched by external magnetic or electric fields.

Several of the novel optical properties observed and predicted in multiferroic materials depend on the inhomogeneous polarization and magnetization across a multiferroic domain boundary. It is known that these domains can be controlled using external magnetic and/or electric fields, and it is this controllable response that makes multiferroics attractive for tunable optical devices. However, exploiting these properties for device applications will require the ability to control the domain wall structure at small length scales. Moreover, nanostructured single phase multiferroics may exhibit very different optical properties than bulk or thin film samples, precisely because of the absence of domain walls. In order to incorporate multiferroics into optical applications, it will be necessary to address at least three fundamental materials questions: Precisely how do the ferroelectric and magnetic domain walls respond to external magnetic and electric fields? Can this behaviour be modulated by patterning on the nanoscale? How does the optical response of multiferroic nanomaterials differ from bulk samples?

Magnetoelectric multiferroics exhibit many optical properties arising from the coexistence of ferroelectrically and magnetically ordered phases. These properties suggest that multiferroic materials could be useful in new types of optical devices. The optical-frequency dielectric response in these systems can be varied by $15 \%$ in a magnetic field, which could be useful for producing highly tunable optical filters. One particularly novel aspect of multiferroics is that the ordered state breaks both spatial inversion symmetry and time reversal symmetry. This results in striking effects in the non-linear optical response, including shifts in the second harmonic generation intensity and the possibility of non-reciprocal refraction through a domain wall. The rich palette of materials properties, and specifically the optical properties, exhibited by multiferroics ensure that these systems will remain an active topic of research for years to come.

\section{Acknowledgements}

This work was supported by NSF CAREER DMR-06044823. I also acknowledge helpful conversations with Chandran Sudakar.

\section{References}

[1] M. Fiebig, "Revival of the magnetoelectric effect," J. Phys. D: Appl. Phys. 38, R123-R152 (2005) [doi:10.1088/0022-3727/38/8/R01]

[2] T. Kimura, T. Goto, H. Shintani, T. Arima, and Y. Tokura, "Magnetic control of ferroelectric polarization," Nature 426, 55-58 (2003) [doi:10.1038/nature02018]

[3] M. Fiebig, Th. Lottermoser, D. Frohlich, A.V. Goltsev, and R.V. Pisarev, "Observation of coupled magnetic and electric domains," Nature 419, 818-820 (2002) [doi:10.1038/nature01077]

[4] H. Zheng, J. Wang, S.E. Lofland, Z. Ma, L. Mohaddes-Ardabili, T. Zhao, L. SalamancaRiba, S.R. Shinde, S.B. Ogale, F. Bai, D. Viehland, Y. Jia, D.G. Schlom, M. Wuttig, A. Roytburd, and R. Ramesh, "Multiferroic $\mathrm{BaTiO}_{3}-\mathrm{CoFe}_{2} \mathrm{O}_{4}$ nanostructures," Science 303, 661-663 (2004) [doi:10.1126/science.1094207]

[5] T. Goto, T. Kimura, G. Lawes, A. P. Ramirez, and Y. Tokura, "Ferroelectricity and giant magnetocapacitance in perovskite rare-earth manganites," Phys. Rev. Lett. 92, 257201 (2004) [doi:10.1103/PhysRevLett.92.257201]

[6] M. Mostovoy, "Ferroelectricity in spiral magnets," Phys. Rev. Lett. 96, 067601 (2006) [doi:10.1103/PhysRevLett.96.067601] 
[7] R.C. Rai, J. Cao, S. Brown, J.L. Musfeldt, D. Kasinathan, D.J. Singh, G. Lawes, N. Rogado, R.J. Cava, and X. Wei, "Optical properties and magnetic-field-induced phase transitions in the ferroelectric state of $\mathrm{Ni}_{3} \mathrm{~V}_{2} \mathrm{O}_{8}$ : Experiments and first principles calculations," Phys. Rev. B 74, 235101 (2006) [doi:10.1103/PhysRevB.74.235101]

[8] Th. Lottermoser, Th. Lonkai, U. Amann, D. Hohlwein, J. Ihringer, and M. Fiebig, "Magnetic phase control by an electric field," Nature 430, 541-544 (2004) [doi:10.1038/nature02728]

[9] S. Ju and G.-Y. Guo, "Colossal nonlinear optical magnetoelectric effects in multiferroic $\mathrm{Bi}_{2} \mathrm{FeCrO}_{6}$, , Appl. Phys. Lett. 92, 202504 (2008) [doi:10.1063/1.2927474]

[10] K. Sawada and N. Nagaosa, "Optical magnetoelectric effect in multiferroic materials: Evidence for a Lorentz force acting on a ray of light," Phys. Rev. Lett. 95, 237402 (2005) [doi:10.1103/PhysRevLett.95.237402] 